\title{
Clinical Outcomes of Spinal Epidural Abscess
}

\author{
HyunJin Ma, Insoo Kim \\ Department of Neurosurgery, Dongsan Medical Center, Keimyung University College of Medicine, Daegu, Korea
}

Objective: The aim of this study is to elucidate the clinical characteristics of patients with spinal epidural abscess (SEA) and demonstrate the risk factors, treatments and neurologic outcomes.

Methods: We retrospectively reviewed the medical records and radiologic images of 35 patients admitted to our department with SEA between March 1987 and April 2011. While we performed decompressive laminectomy and abscess drainage on 19 patients $(54.3 \%)$, and 16 patients $(45.7 \%)$ initially received conservative therapy with antibiotics alone. Medical Research Council (MRC) scale was applied to estimate results objectively.

Results: The neurological outcome data showed improved MRC scale from 14 (40\%) patients. $13(37.1 \%)$ patients showed unchanged MRC scale and $8(22.9 \%)$ patients revealed worsened MRC scale at the time of discharge. The patients with surgical treatment showed more improved MRC scale than the patients with conservative treatment and this was statistically significant $(p=0.001)$ on univariate analysis. Initially, patients with decreased MRC scale and rapidly progressing neurological deficit underwent emergency surgery within 24 hours. Patients with intact MRC scale and minor neurological deficit received delayed surgery or conservative management with antibiotics. Among 19 patients those who experienced emergent operations within 24 hours showed better prognosis than those who underwent delayed operations after 24 hours.

Conclusion: Surgical treatment is the modality of choice in patients with SEA and urgent surgery especially is indicated in patients with neurological deficits. And early surgery is more effective in neurological improvements than delayed surgery and conservative management.

Key Words: Epidural abscess $\cdot$ Spine $\cdot$ Surgery

\section{INTRODUCTION}

Spinal epidural abscess (SEA) is a relatively rare disease but frequently, emergent surgery is needed ${ }^{29,12,18)}$. Their incidence is on the rise because of the various factors such as the increase of the elderly population, intravenous drug use, epidural steroid injection for pain control, epidural anesthesia and development of imaging methods such as computed tomography (CT) and magnetic resonance imaging (MRI).

With the development of diagnostic radiological evaluations such as CT or MRI, rapid and accurate diagnosis has been available, leading to the significant improvement of mortality and morbidity. However, neurological complete recovery rate and mortality were reported to be still $16 \%$ and $41-47 \%$, respe-

\footnotetext{
- Received: February 1, 2012 • Revised: February 27, 2012

- Accepted: March 17, 2012

Corresponding Author: Insoo Kim, MD, PhD

Department of Neurosurgery, Dongsan Medical Center, 56 Dalseong-ro, Jung-gu, Daegu, 700-712, Korea

Tel: +82-53-250-7730, Fax: +82-53-250-7356

E-mail: neurokim@dsmc.or.kr
}

ctively despite appropriate treatments ${ }^{4,15}$.

The aim of this study is to describe the clinical characteristics of 35 patients with SEA and to disclose the risk factors, treatments and neurologic outcomes.

\section{MATERIAL AND METHODS}

This study was conducted on 35 patients who had been diagnosed with SEA at the department of neurosurgery in our hospital for 24 years from April 1987 to April 2011. We excluded osteomyelitis or discitis without true epidural collection and SEA due to tuberculous spondylitis. We retrospectively reviewed the following data from the medical records including age, gender, clinical manifestation, laboratory data, imaging finding such as CT and MRI and neurological outcome data at the time of discharge. The patients who underwent either emergent operation within 24 hours or delayed surgery after 24 hours, or conservative management were dealt case by case. Emergency surgeries within 24 hours were conducted when patients had neurological deficit or neurological deterioration and 16 patients received antibiotics treatment alone 
without surgery. Neurological outcome and clinical outcome were classified by comparing the MRC scale at the time of diagnosis and at the time of discharge. To elucidate improved to worsened outcomes of patients by treatment methods, we used chi square test.

\section{RESULTS}

On admission to our department, there were 22 males (62.9 $\%)$ and 13 females (37.1\%). The mean age of the patients at diagnosis was 64 years old. The presented symptoms were neck pain or back pain in 12 cases (34.3\%), radiating pain in 22 cases (62.9\%), and neurologic deficits such as motor weakness and sensory change in 28 cases (80\%). Fever upon hospitalization was shown in 10 cases (28.6\%), and the mean number of white blood cell (WBC) was shown to be 9,886 $\pm 3,776$ cells/mL. Leukocytosis was observed in only 7 cases (20\%). The mean C-reactive protein (CRP) was shown to be $4.94 \pm 4.05$ $\mathrm{mg} / \mathrm{L}$, and the increased CRP was shown in 19 cases (54.3\%).

Risk factors predisposing to spinal epidural abscess were found in 34 patients. Non-spinal infections were the most common cause of SEA. Among them, paravertebral abscess was the utmost cause, and skin and soft tissue abscess was followed by it. In addition, risk factors were found in spinal procedure such as epidural injection for pain control, acupuncture and previous spine surgery after spinal trauma. Risk factors such as diabetes mellitus, liver disease, and cancer were also found. No risk factor was found in 1 case (Table 1).

The location of epidural abscess collection was in the cervical spine in 4 patients, thoracic in 8 , thoracolumbar in 3 , lumbar in 19, and lumbosacral in 1 patient. The extents of spinal epidural abscesses ranged from 1 to 6 segments, and the mean was 2.7 segments. The spinal epidural collection was dorsal segment to the thecal sac in 4 cases, ventral segment to the thecal sac in 24 cases, and circumference to the thecal sac in 7 cases (Table 2).

Gadolinium enhanced MRI was performed in 34 cases and CT was conducted in 1 case when MRI was unavailable. MRI showed iso or low signal intensity in T1 weighted image (T1WI), whereas it exhibited high signal intensity in T2 weighted image (T2WI). After gadolinium administration, rim enhancement was found in 22 cases, whereas heterogeneous enhancement was found in 12 cases (Fig. 1).

The initial MRC scale of patients was $1 / 5$ in 5 patients, 2/5 in 1 patient, $3 / 5$ in 3 patients, $4 / 5$ in 18 patients, and 5/5 in 8 patients. And the post treatment MRC scale was $1 / 5$ in 1 patient, $2 / 5$ in 4 patients, $3 / 5$ in 6 patients, $4 / 5$ in 6 patients, and $5 / 5$ in 18 patients (Table 3).

Once SEA was diagnosed in the patients, an immediate treat-
Table 1. Associated predisposing factor

\begin{tabular}{lc}
\hline \multicolumn{1}{c}{ Risk factor } & Number of patients (\%) \\
\hline Paravertebral abscess & $12(35)$ \\
Skin \& soft tissue infection & $5(14)$ \\
Perirenal abscess & $1(3)$ \\
Endocarditis & $1(3)$ \\
Epidural injection & $2(6)$ \\
Acupuncture & $3(8)$ \\
Previous spine surgery & $1(3)$ \\
Vertebral body fracture & $3(8)$ \\
Psoas muscle contusion & $1(3)$ \\
Diabetes mellitus & $1(3)$ \\
Liver disease & $3(8)$ \\
Cancer & $1(3)$ \\
No risk & $1(3)$ \\
\hline
\end{tabular}

Table 2. Level and location of spinal epidural abscess

\begin{tabular}{lccc}
\hline \hline Level \& location & $\begin{array}{c}\text { Anterior to } \\
\text { thecal sac }\end{array}$ & $\begin{array}{c}\text { Posterior to } \\
\text { thecal sac }\end{array}$ & Circumference \\
\hline Cervical & 3 & 0 & 1 \\
Thoracic & 6 & 1 & 1 \\
Thoracolumbar & 2 & 1 & 0 \\
Lumbar & 12 & 2 & 5 \\
Lumbosacral & 1 & 0 & 0 \\
\hline
\end{tabular}

Table 3. MRC scale of patients at pre- and post treatment

\begin{tabular}{ccc}
\hline \hline MRC scale & Pre-treatment & Post-treatment \\
\hline $0 / 5$ & 0 & 0 \\
$1 / 5$ & 5 & 1 \\
$2 / 5$ & 1 & 4 \\
$3 / 5$ & 3 & 6 \\
$4 / 5$ & 17 & 8 \\
$5 / 5$ & 9 & 16 \\
\hline
\end{tabular}

MRC: Medical Research Council

ment was initiated to all SEA patients. Laminectomy, decompressive debridement, abscess drainage, or spinal fusion was performed in the patients with minor or severe neurologic deficit, and spinal fusion was performed in the patients with instability or spinal cord compression in the imaging study even if there were no neurologic deficit. Laminectomy was performed in 15 cases and laminectomy with spinal fusion was performed in 4 cases. Antibiotics alone therapy was performed in patients with no neurologic deficit and minimal spinal cord compression in the imaging study.

All SEA patients underwent antibiotics treatments. Empirical antibiotics covering Staphylococcus aureus was initially used and was changed with sensitive antibiotics according to the culture of abscess. Causative pathogens were identified in 13 cases (37.1\%) in the abscess culture, of which Staphylo- 

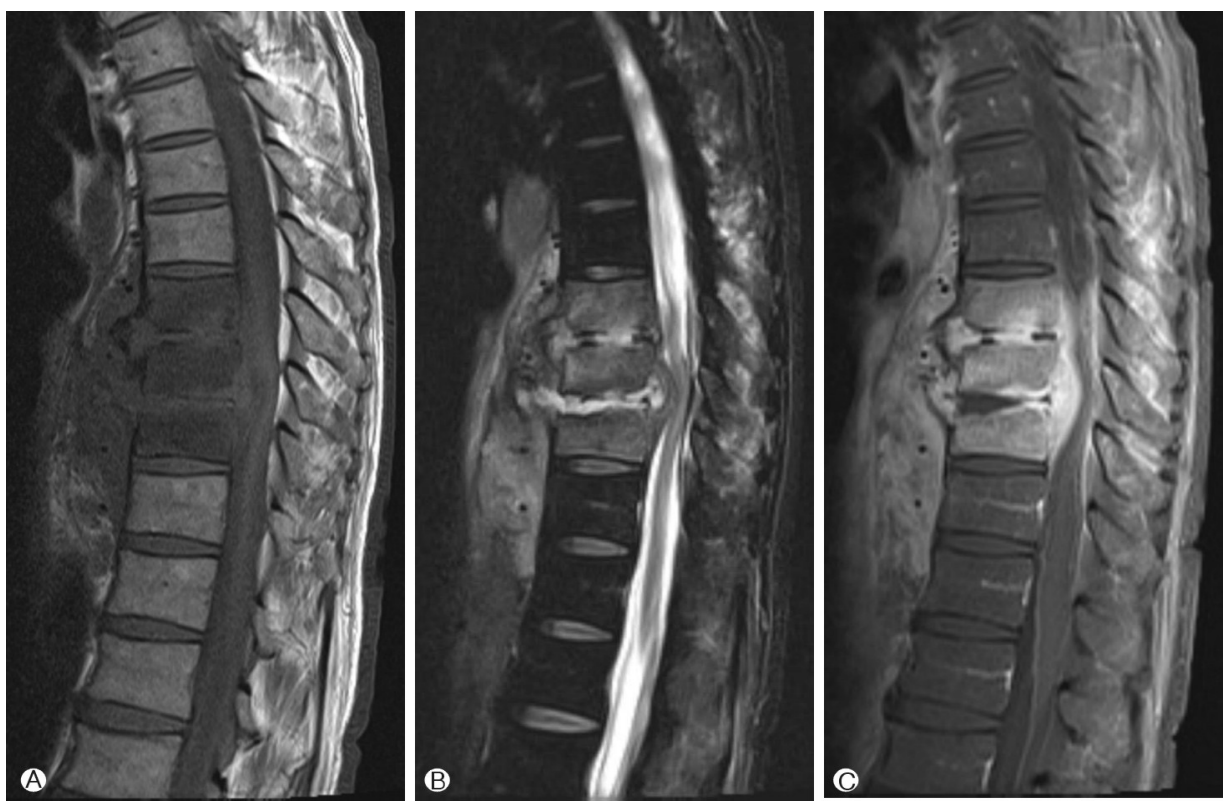

Fig. 1. The epidural collection on $\mathrm{T} 8$ to $\mathrm{T10}$ is isointense on $\mathrm{Tl}$-weighted image (A), hyperintense on T2weighted image $(B)$ and rim enhanced after gadolinium administration (C)
Table 4. The outcomes of surgical and conservative cases

\begin{tabular}{lccc}
\hline \hline & $\begin{array}{c}\text { Improved } \\
\text { MRC scale } \\
(\mathrm{n}=14)\end{array}$ & $\begin{array}{c}\text { Unchanged } \\
\text { MRC scale } \\
(\mathrm{n}=13)\end{array}$ & $\begin{array}{c}\text { Worsened } \\
\text { MRC scale } \\
(\mathrm{n}=8)\end{array}$ \\
\hline Emergent operation & 10 & 1 & 0 \\
Delayed operation & 3 & 2 & 3 \\
Conservative treatment & 1 & 10 & 5 \\
\hline
\end{tabular}

MRC: Medical Research Council

coccus aureus was found in 7 cases (53.8\%). Streptococcus species was found in 2cases (15.3\%), klebsiella, and Burholderia cepacia were found in one case (7.6\%) each. A positive result in the blood culture was shown in 8 cases (22.9\%), and Staphylococcus aureus was most frequently found. For the period of antibiotics use, an intravenous antibiotic was used for 4-8 weeks while monitoring WBC count, erythrocyte sedimentation rate (ESR) and CRP, and an oral antibiotic was additionally used in some patients for 2-3 weeks more.

14 cases (40\%) showed improvement of MRC scale and 13 cases (37.1\%) were unchanged. Nevertheless, MRC scale was worsened in 8 cases (22.9\%). Of the 14 cases showing improvement, 10 patients underwent the emergent surgery within 24 hours and 3 patients had the delayed surgery over 24 hours and 1 patient received conservative treatment with antibiotics alone. Among 13 patients who showed no change in MRC scale, 1 patient underwent the emergency surgery, 2 patients experienced the delayed surgery and 10 patients underwent conservative treatment with antibiotics alone. Of the 8 patients who showed worsened neurologic deficit, no patient received emergency surgical treatment, 3 patients underwent
Table 5. Univariate analysis for outcome between operation and conservative management

\begin{tabular}{|c|c|c|c|c|}
\hline & $\begin{array}{c}\text { Improved } \\
\text { MRC scale } \\
(n=14)\end{array}$ & $\begin{array}{l}\text { Unchanged } \\
\text { MRC scale } \\
(n=13)\end{array}$ & $\begin{array}{c}\text { Worsened } \\
\text { MRC scale } \\
(n=8)\end{array}$ & p-value \\
\hline Operation & 13 & 3 & 3 & \\
\hline $\begin{array}{l}\text { Conservative } \\
\text { manage }\end{array}$ & 1 & 10 & 5 & 0.001 \\
\hline
\end{tabular}

Table 6. Univariate analysis for initial MRC scale of the patients and treatment method

\begin{tabular}{lcccc}
\hline \hline & $\begin{array}{c}\text { Decreased MRC } \\
\text { scale }(\mathrm{n}=26)\end{array}$ & $\begin{array}{c}\text { Intact MRC } \\
\text { scale }(\mathrm{n}=9)\end{array}$ & p-value \\
\hline Emergency operation & 11 & 0 & \\
Delayed operation & 7 & 1 & 0.009 \\
Conservative manage & 8 & 8 & \\
\hline
\end{tabular}

$p<0.05$. MRC: Medical Research Council

delayed surgical treatment and 5 patients experienced antibiotic treatment (Table 4). Between operative and conservative management, operation, regardless of emergency or delay, was more effective than conservative management to improve neurological deficit measured by MRC scale (Table 5). Initially, patients with decreased MRC scale and rapidly progressing neurological deficit underwent emergency surgery within 24 hours. Patients with intact MRC scale and minor neurological deficit got delayed surgery over 24 hours or conservative management with antibiotics (Table 6). Among 19 patients those 
Table 7. Univariate analysis for outcomes of the patients with emergency operation within 24 hours or delayed operation over 24 hours

\begin{tabular}{lcccc}
\hline \hline & $\begin{array}{c}\text { Improved } \\
\text { MRC scale } \\
(n=13)\end{array}$ & $\begin{array}{c}\text { Unchanged } \\
\text { MRC scale } \\
(n=3)\end{array}$ & $\begin{array}{c}\text { Worsened } \\
\text { MRC scale } \\
(n=7)\end{array}$ & p-value \\
\hline $\begin{array}{l}\text { Emergency } \\
\text { operation }\end{array}$ & 10 & 1 & 0 & \\
$\begin{array}{l}\text { Delayed } \\
\text { operation }\end{array}$ & 3 & 2 & 3 & 0.033 \\
\hline$p<0.05$. MRC: Medical & Research Council & & \\
\hline
\end{tabular}

Table 8. Univariate analysis for outcome between culture positive and negative

\begin{tabular}{|c|c|c|c|c|}
\hline & $\begin{array}{c}\text { Improved } \\
\text { MRC scale } \\
(n=14)\end{array}$ & $\begin{array}{c}\text { Unchanged } \\
\text { MRC scale } \\
(n=13)\end{array}$ & $\begin{array}{c}\text { Worsened } \\
\text { MRC scale } \\
(n=8)\end{array}$ & p-value \\
\hline Culture positive & 9 & 4 & 0 & \multirow{2}{*}{0.009} \\
\hline Culture negative & 5 & 9 & 8 & \\
\hline
\end{tabular}

who underwent emergent operation within 24 hours showed better prognoses than those who received delayed operation after 24 hours. This result was statistically significant $(\mathrm{p}=$ 0.033) (Table 7). 13 patients who had positive culture revealed more improvement of MRC scale than the patients with negative culture $(\mathrm{p}=0.009)$ (Table 8$)$.

\section{DISCUSSION}

SEA mainly occurs in patients aged from 30 to 70 years old and a very rare disease with a prevalence of $1-2$ persons $/ 10,000$ patients before 1990s. However, it has been recently increasing with a prevalence of $10-12$ persons/ 10,000 patients due to increased elderly population, intravenous drug abuse, patient controlled analgesia, and spinal anesthesia for pain treatment as well as decreased immune response caused by various reasons $^{1,10,22)}$.

In our series, a specific co-morbid condition with spinal epidural abscess was non-spinal infection in 19 cases (55\%), spinal procedure in 6 cases (17\%), diabetes mellitus in 1 case (3\%), spinal trauma in 4 cases (11\%), liver disease in 3 cases $(8 \%)$, cancer in 1 case (3\%) and no risk in 1 case (3\%).

Three main mechanisms of SEA are as follows: First, results from hematogenous dissemination of distant infectious foci such as urinary tract infection, pneumonia, and laryngitis, account for the highest proportion. Second, infections are directly transmitted from adjacent structures such as vertebral osteomyelitis, spondylodiscitis, and perispinal mass abscess.
Third, infections are transmitted via the hands such as spinal surgery, epidural injection therapy, and spinal anesthesia ${ }^{7,211}$.

Staphylococcus aureus has been known to be the most common causative pathogen accounting for 42-84\%, and Streptococcal strains and gram-negative bacteria are followed by Staphylococcus aureus ${ }^{4)}$. In this study, Staphylococcus aureus was shown to be the most frequently found causative pathogen. However, due to the early use of antibiotics prior to bacteria culture and insufficient tissue for bacteria culture, the unidentified causative pathogen and the negative result in the blood culture has been known to account for $24.4 \%$ and $50 \%$, respectively ${ }^{6,8,19)}$. In this study, the successful bacteria culture in the abscess and in the blood was shown in 13 cases (37.1\%) and in 8 cases (22.9\%), respectively.

SEA most frequently occurs in the thoracic region, and then in the lumbar region, whereas it has a lowest prevalence in the cervical region. The higher prevalence of SEA in the thoracic region is attributable to larger epidural fat tissue and space. The lower prevalence of SEA in the cervical region is attributable to the limited epidural fat tissue and space ${ }^{7,11)}$. In this study, SEA was shown to occur in the lumbar regions in 19 cases, almost half of all the presented cases (54.2\%).

MRI with gadolinium enhance is the most commonly used radiologic diagnostic method in these days. CT has an advantage of identifying small bone destruction or paraspinal abscess, but has disadvantages of difficulties in identifying the small amount of abscess and accurately assessing the spreading level of abscess into the adjacent area due to low contrast among the tissues ${ }^{11)}$. MRI is a noninvasive diagnostic method and is easily used in patients for diagnosis. In addition, the distribution of epidural abscess can be accurately identified via $\mathrm{MRI}^{10,13,20)}$. Typical findings include iso or low signal intensity in T1WI and heterogeneous high signal intensity in $\mathrm{T} 2 \mathrm{WI}^{3)}$. As MRI has a disadvantage of a difficulty in assessing the change of the concurrent adjacent bone structure, simple X-ray or CT is conducted with MRI to accurately assess the state of the lesions ${ }^{14)}$.

Treatment of SEA is comprised of immediate decompression and surgical drainage, and appropriate administration of antibiotics before and after the surgery ${ }^{3,10,14)}$. Through immediate decompression and surgical drainage, the neurologic deficit can be repaired as well as pathological diagnosis, and the identification of causative pathogens can be achieved ${ }^{15,20)}$. Operation strategy should be determined according to abscess location, concurrent osteomyelitis, and vertebral body destruction. Laminectomy is the most commonly used in the case of abscess located in the posterior spine without lesions in the vertebral body. In addition, in the cases of compression rate of the vertebral body more than $50 \%$, defected posterior spinal structure and the kyphotic angle of more than $20^{\circ}$, internal 
fixation with autologous bone graft is required ${ }^{16,17)}$.

Because most infections are caused by Staphylococcus aureus, empirical antibiotics covering Staphylococcus aureus was initially used and was changed with sensitive antibiotics according to the sensitivity. The 4-week period of antibiotics use is generally recommended in the case of abscess without pyogenic spondylitis. The 8-week period of antibiotics use is recommended in the case of abscess with pyogenic spondylitis. Furthermore, an oral antibiotics is additionally used for extra 2-3 weeks while monitoring ESR and $\mathrm{CRP}^{4,8}$.

In the past, a mortality of $100 \%$ was reported in the case of no surgical treatment, and a mortality of $32 \%$ was reported in the case of surgical treatment, which showed high morbidity and mortality ${ }^{11)}$. At present, a neurologically complete recovery rate of $41-47 \%$ and a mortality of $16 \%$ are still reported even in the case of appropriate treatments ${ }^{4,15}$. In general, there were no statistical differences between groups in age, gender, location of abscess, number of involved vertebral levels or risk factors. Complete recovery can be expected in the cases of no paraplegia before operation or paraparesis period of 36 hours or less. However, neurological recovery cannot be achieved in the case of paraplegia of more than 48 hours as neurological paraplegia causes not only mechanical decompression, but also circulatory disturbance due to thrombosis caused by poor inflammatory response, thereby causing spinal neurologic deficit ${ }^{5,11,20)}$. Our study suggests that neurological improvement in spinal epidural abscess is more easily achieved via decompressive laminectomy or abscess drainage than conservative treatment after neurological deterioration.

Poor prognoses are expected in the cases of intraspinal high signal intensity in T1WI, thrombocytopenia, increased ESR, increased CRP or pancytopenia in hematologic test, pure abscess with no granulation tissue, and antibiotics treatment alone without surgical treatment ${ }^{4,6,8}$. In particular, prognoses are poorer in the case of thoracic region than in the cases of cervical or lumbar region. This is likely to be attributable to rapid neurologic deficit due to narrow spinal subarachnoid space and poor circulation ${ }^{14)}$.

In this study, better recovery of neurologic deficit was shown in the patients with surgical treatment than in the patients with antibiotics treatment alone. Patients who had decreased MRC scale at diagnosis, had more incidence to receive surgery than who had intact MRC scale. We had decompressive surgery and abscess drainage to patients with decreased MRC scale at diagnosis if condition of patient was not excessively bad for operation. In the patients with surgery, better prognoses were shown in the patients with emergent surgery within 24 hours than in the patients with delayed operation after 24 hours. Each patient had its own reasons for treatment and as our study is retrospective, we could not compare with the other treatments. Thus, it is insufficient to conclude early surgery is better than the other treatments and it is the limitation of our study. However, good outcomes can be expected if decompression and surgical drainage are conducted immediately after diagnosing SEA. And the improvement of neurological symptoms can be expected through emergency surgery particularly in the patients with neurological deficit with decreased MRC scale.

\section{CONCLUSION}

SEA is a rare disorder that can cause neurological deterioration or death if it is not recognized or treated early. Therefore, SEA should be considered in patients with back or radiating pain, motor weakness or sensory change. And if SEA is diagnosed, although the patients have minor neurological deficit or no motor weakness, early surgical removal of SEA should be considered. We can expect desirable outcomes through immediate surgical drainage and decompression when SEA is diagnosed. In particular, satisfactory outcomes can be achieved from emergent surgery within 24 hours from diagnosis of SEA even if neurological deficit is observed at the time of diagnosis.

\section{REFERENCES}

1. Abel R, Von Baum H: Multilevel epidural 1 abscess formation with paraplegia in a healthy 33-year-old man caused by Staphylococcus aureus (MSSA). Infection 31:359-361, 2003

2. Ahl T, Hedstrom M, von Heijne A, Stiernstedt SH: Acute spinal epidural abscess without concurrent spondylodiscitis. Successful closed treatment in 10 cases. Acta Orthop Scand 70:199202, 1999

3. Akalan N, lan T: Infection as a cause of spinal cord compression: A review of 36 spinal epidural abscess cases. Acta Neurochir (Wien) 142:17-23, 2000

4. Curry WT Jr., Hoh BL, Amin-Hanjani S, Eskandar EN: Spinal epidural abscess: clinical presentation, management, and outcome. Surg Neurol 63:364-371, 2005

5. Hadjipavlou AG, Katonis PK, Gaitanis IN, Muffoletto AJ, Tzermiadianos MN, Crow W: Percutaneous transpedicular disectomyand drainage in pyogenic spondylodiscitis. Eur Spine J 13:707-713, 2004

6. Hadjipavlou AG, Mader JT, Necessary JT, Muffoletto AJ: Hematogenous Pyogenic spinal infections and their surgical management. Spine 5:1668-1679, 2000

7. Hsieh PC, Wienecke RJ, O'Shaughnessy BA, Koski TR, Ondra SL: Surgical strategies for vertebral osteomyelitis and epidural abscess. Neurosurg Focus 17:E4, 2004

8. Jung YJ, Kim SW, Chang CH, Kim SH, Kim OL, Cho SH: Prognostic factors of pyogenic Spinal infections. J Korean 
Neurosurg Soc 38:445-449, 2005

9. Khanna RK, Malik GM, Rock JP, Rosenblum ML: Spinal epidural abscess: evaluation of factors influencing outcome. Neurosurgery 39:958-964, 1996

10. Khan SH, Hussain MS, Griebel RW, Hattingh S: Title comparison of primary and secondary spinal epidural abscesses: A retrospective analysis of 29 cases. Surg Neurol 59:28-33, 2003

11. Kim H, Oh SH, Choi IS, Bak KH, Kim YS, Kim CH, et al: Acute panspinal epidural abscess. J Korean Neurosurg Soc 28:392-327, 1999

12. Kim JS, Sung KH, Cho YW, Kim KH, Bae SD: Extensive Spinal Epidural Abscess: The Usefulness of Minimal Invasive Surgical Technique using Epidural Irrigation Catheter. Korean J Spine 8:221-224, 2011

13. Kowalski TJ, Layton KF, Berbari EF, Steckelberg JM, Huddleston PM, Wald JT, et al: Follow-Up MR imaging in Patients with Pyogenic Spine Infections: Lack of Correlation with Clinical Features. A JNR Am J Neuroradiol 28:693-699, 2007

14. Lee JK, Kim SH, Kim ES, Kim TS, Jung S, Kim JH, et al: Surgical Treatment of the Spontaneous Spinal Epidural Abscess. J Korean Neurosurg Soc 29:1037-1042, 2000

15. Mann S, Schutze M, Sola S, Piek J: Nonspecific pyogenic spondylodiscitis: clinical manifestations, surgical treatment, and out- come in 24 patients. Neurosurg Focus 17:E3, 2004

16. Muzii VF, Mariottini A, Zalaffi A, Carangelo BR, Palma L: Cervical spine epidural abscess: experience with microsurgical treatment in Eight cases. J Neurosurg Spine 5:392-397, 2006

17. Nakase H, Matsuda R, Tamaki R, Tei R, Park YS, Sakaki T: Two-stage management for vertebral osteomyelitis and epidural abscess: technical note. Neurosurgery 58:E1219, 2006

18. Nussbaum ES, Rigamonti D, Standiford H, Numaguchi Y, Wolf AL, Robinson WL: Spinal epidural abscess: a report of 40 cases and review. Surg Neurol 38:225-231, 1992

19. Ogden AT, Kaiser MG: Single-stage debridement and instrumentation for pyogenic spinal infections. Neurosurg Focus 17:E5, 2004

20. Wilhelm K, Michael M, Lothar M, Rudolf T, Armin T: Epidural spinal infection: Variability of clinical and magnetic resonance imaging finding. Spine 22:544-550, 1997

21. Yamada K, Ohnari M, Takahashi R, Taniguchi J, Hamatani $\mathrm{K}$ : Pyogenic spondylitis and epidural abscess distant from the site of Continuous epidural block in a patient with postherpetic neuralgia. J Anesth 15:176-178, 2001

22. Yoo YS, Lee JC, Son EI, Kim DW, Yim MB, Lee CY, et al: Review of 10 cases of pyogenic epidural abscess of the spine. J Korean Neurosurg Soc 25:1270-1276, 1996 